1 Hacettepe Journal of Mathematics and Statistics

$\bigcap$ Volume 45 (6) (2016), 1719-1728

\title{
Values sharing results on $q$-difference and derivative of meromorphic functions
}

\author{
Kai Liu* ${ }^{* \dagger}$ and Tingbin Cao ${ }^{\ddagger}$
}

\begin{abstract}
In this paper, we mainly deal with the problem that $f(q z)$ and $f^{\prime}(z)$ share common values. One of the purpose is to explore whether the classical uniqueness results remain valid or not by considering some uniqueness theorems on $f(q z)$ and $f^{\prime}(z)$ sharing common values. Some examples and remarks are given to show that our results are sharp in certain senses. We also consider the entire solutions of the equation $f^{\prime}(z)=f(q z)$, which is important for the uniqueness results.
\end{abstract}

Keywords: Value sharing; $q$-difference; derivative; order.

2000 AMS Classification: 30D35, 39A05, 34M05.

Received : 10.04.2014 Accepted : 17.02.2016 Doi : 15672/HJMS.20164515998

\section{Introduction}

We use the standard symbols and fundamental results of Nevanlinna theory $[7,10,18]$. A meromorphic function $f(z)$ means meromorphic in the complex plane $\mathbb{C}$. If $f-a$ and $g-a$ have the same zeros, then we say that $f$ and $g$ share the value $a I M$ (ignoring multiplicities). If $f-a$ and $g-a$ have the same zeros with the same multiplicities, then $f$ and $g$ share the value $a C M$ (counting multiplicities).

Recall a classical result given by Rubel and Yang [16] as follows.

Theorem A. Let $f(z)$ be a non-constant entire function. If $f(z)$ and $f^{\prime}(z)$ share two values $a, b \in \mathbb{C} C M$, then $f^{\prime}(z)=f(z)$.

Many improvements on Theorem A were investigated afterwards. For example, $f^{\prime}(z)$ was improved to $f^{(k)}(z)$ or differential polynomials of $f(z)$, the condition $C M$ was reduced

\footnotetext{
*Department of Mathematics, Nanchang University, Nanchang, Jiangxi, 330031, P. R. China, Email: liukai418@126.com, liukai@ncu.edu.cn

†Corresponding Author.

†Department of Mathematics, Nanchang University, Nanchang, Jiangxi, 330031, P. R. China, Email: tbcao@ncu.edu.cn
} 
to $I M$, an entire function $f(z)$ was extended to a meromorphic function, and so on. We only recall the following result given by Mues-Steinmetz [12].

Theorem B. Let $f(z)$ be a non-constant entire function. If $f(z)$ and $f^{\prime}(z)$ share two values $a, b \in \mathbb{C} I M$, then $f^{\prime}(z)=f(z)$.

Recently, Qi, Liu and Yang [14] considered the problem that $f(z)$ and $f(q z)$ share common values, where $f(z)$ is a zero-order meromorphic function and $|q|=1$. One of the results can be stated as follows.

Theorem C. [14, Theorem 1.1] Let $f(z)$ be a zero-order meromorphic function and $a_{1}, a_{2}, a_{3} \in \mathbb{C} \cup\{\infty\}$ be three distinct values. If $f(z)$ and $f(q z)$ share $a_{1}, a_{2} C M$ and $a_{3}$ $I M$, then $f(z)=f(q z)$.

Here, two remarks are given to show that the conditions of Theorem $\mathrm{C}$ are indispensable, which are not considered in [14].

1.1. Remark. Theorem $\mathrm{C}$ is not valid for meromorphic functions with finite order, which can be seen by the following two examples.

1.1. Example. If $f(z)=e^{z}$ and $q=-1$, then $f(z)$ and $f(q z)$ share $0,1, \infty C M$, but $f(z) \neq f(q z)$.

1.2. Example. If $f(z)=e^{z^{2}}$ and $q=i$, then $f(z)$ and $f(q z)$ share $0,1, \infty C M$, but $f(z) \neq f(q z)$.

1.2. Remark. The condition $a_{1}, a_{2} C M$ and $a_{3} I M$ can not be reduced to $a_{1}, a_{2} C M$ in Theorem $\mathrm{C}$, which can be seen by the following example.

1.3. Example. If $f(z)=\frac{2 z}{(z+1)^{2}}$ and $q=-1$, then $f(q z)=\frac{-2 z}{(1-z)^{2}}$. We know that $f(z)$ and $f(q z)$ share $0,1 C M$, but $f(z) \neq f(q z)$.

However, if $f$ is an entire function with zero-order, then the conditions of Theorem $\mathrm{C}$ can be reduced as follows.

Theorem D. [14, Theorem 1.2] Let $f$ be a zero-order entire function and $a_{1}, a_{2} \in \mathbb{C}$ be two distinct values. If $f(z)$ and $f(q z)$ share $a_{1}$ and $a_{2} I M$, then $f(z)=f(q z)$.

Noticing the above four theorems, Theorem A and Theorem B are related to the value sharing problem on $f(z)$ and $f^{\prime}(z)$, Theorem $\mathrm{C}$ and Theorem $\mathrm{D}$ are related to the value sharing problem on $f(z)$ and $f(q z)$. An interesting problem is what can we get if $f^{\prime}(z)$ and $f(q z)$ share common values, where $q$ is a non-zero constant. Some related results can be found in Section 3. Some results on the zeros distribution of $q$-difference differential polynomials of different types and uniqueness results can be seen in Section 4 .

\section{The entire solutions of $f^{\prime}(z)=f(q z)$}

As we all know that the differential equation $f^{\prime}(z)=f(z)$ implies that $f(z)=A e^{z}$, where $A$ is a constant. Before considering the value sharing problem on $f(q z)$ and $f^{\prime}(z)$, we should consider the solutions properties of the $q$-difference differential equation

$$
f^{\prime}(z)=f(q z),
$$

where $q$ is a non-zero constant. Obviously, the non-trivial entire solutions of (2.1) should be transcendental. Using the theory of series, we obtain the next result.

2.1. Theorem. The non-trivial entire solutions of (2.1) must be have the form

$$
f(z)=\sum_{n=0}^{+\infty} \frac{a_{0}}{n !} q^{\frac{n(n-1)}{2}} z^{n},
$$

where $a_{0}$ is a free complex parameter. 
Proof. Let $f(z)=a_{0}+a_{1} z+a_{2} z^{2}+\cdots+a_{n} z^{n}+\cdots$. Thus

$$
f(q z)=a_{0}+a_{1} q z+a_{2}(q z)^{2}+\cdots+a_{n}(q z)^{n}+\cdots
$$

and

$$
f^{\prime}(z)=a_{1}+2 a_{2} z+\cdots+n a_{n} z^{n-1}+\cdots .
$$

By comparing with the coefficients of (2.2) and (2.3), we get

$$
\begin{gathered}
a_{1}=a_{0} q^{0}, \\
a_{2}=\frac{a_{0}}{2} q^{1}, \\
a_{3}=\frac{a_{0}}{3 \times 2} q^{1+2}, \\
a_{4}=\frac{a_{0}}{4 \times 3 \times 2} q^{1+2+3}, \\
a_{5}=\frac{a_{0}}{5 \times 4 \times 3 \times 2} q^{1+2+3+4}, \\
a_{6}=\frac{a_{0}}{6 \times 5 \times 4 \times 3 \times 2} q^{1+2+3+4+5}, \ldots
\end{gathered}
$$

Using mathematical induction, we get $f(z)$ should have the form

$$
f(z)=\sum_{n=0}^{+\infty} \frac{a_{0}}{n !} q^{\frac{n(n-1)}{2}} z^{n} .
$$

2.1. Remark. As we all know that if $g(z)=\sum_{n=0}^{\infty} a_{n} z^{n}$ is an entire function, the order's expression

$$
\rho(g)=\varlimsup_{n \rightarrow \infty} \frac{n \log n}{\log \frac{1}{\left|a_{n}\right|}} .
$$

Thus, we conclude that $\rho(f)=0$ if $|q| \neq 1$ and $\rho(f)=1$ if $|q|=1$ in Theorem 2.1. Obviously, if $q=1$, then $f(z)=\sum_{n=0}^{+\infty} \frac{a_{0}}{n !} z^{n}=a_{0} e^{z}$.

\section{Some results on $f(q z)$ and $f^{\prime}(z)$ share common values}

Let us recall the classical results in the uniqueness theory of meromorphic functions, the five-point, resp. four-point, theorems due to Nevanlinna [15].

The five-point theorem. If two meromorphic functions $f, g$ share five distinct values in the extended complex plane $I M$, then $f \equiv g$.

The four-point theorem. If two meromorphic functions $f, g$ share four distinct values in the extended complex plane $C M$, then $f \equiv T(g)$, where $T$ is a Möbius transformation.

If the meromorphic function $g$ has a special relationship with $f$, then the number five or four can be reduced. For example, considering the value sharing problem on $f(z)$ and $f(z+c)$ [8, Theorem 2] or $f(z)$ and $f(q z)$ [14, Theorem 1.1], the number is three. Before stating our results, we need the following lemma [18, Theorem 2.17].

3.1. Lemma. Let $f$ and $g$ be non-constant meromorphic functions with the order less than one. If $f$ and $g$ share 0 and $\infty C M$, then there exists a non-zero constant $K$ satisfying $f=K g$.

Let $f_{1}=\frac{f-a_{1}}{f-a_{2}}$ and $g_{1}=\frac{g-a_{1}}{g-a_{2}}$. If $f$ and $g$ share $a_{1}$ and $a_{2} C M$, then $f_{1}$ and $g_{1}$ share $0, \infty C M$, thus we have $\frac{f-a_{1}}{f-a_{2}}=k \frac{g-a_{1}}{g-a_{2}}$. 
3.2. Theorem. Let $f$ be a meromorphic function with order $\rho(f)<1$ and let $a_{1}, a_{2} \in$ $\mathbb{C} \cup\{\infty\}$ and $a_{3} \in \mathbb{C}$ be three distinct values. If $f(q z)$ and $f^{\prime}(z)$ share $a_{1}, a_{2} C M$ and $a_{3}$ $I M$, then $f^{\prime}(z)=f(q z)$.

Proof. If $a_{1}, a_{2}, a_{3} \in \mathbb{C}$. Let $F(z)=\frac{f^{\prime}(z)-a_{1}}{f^{\prime}(z)-a_{2}} \cdot \frac{a_{3}-a_{2}}{a_{3}-a_{1}}$ and $G(z)=\frac{f(q z)-a_{1}}{f(q z)-a_{2}} \cdot \frac{a_{3}-a_{2}}{a_{3}-a_{1}}$. Thus, we have $F(z)$ and $G(z)$ share $0, \infty C M$ and $1 I M$. Since that $F(z)$ and $G(z)$ are meromorphic functions with $\rho(f)<1$, then $F(z)=k G(z)$ follows from Lemma 3.1. If the value 1 is not the Picard exceptional value, then $k=1$, thus $F(z)=G(z)$. If the value 1 is the Picard exceptional value, we have $\frac{F(z)-1}{G(z)-1}$ has no zeros and poles. Hence, we have $\frac{F(z)-1}{G(z)-1}=C$, which implies that $k=1$, thus $F(z)=G(z)$. We conclude that $f^{\prime}(z)=f(q z)$

If one of $a_{1}, a_{2}$ is $\infty$, without loss of generality, we suppose that $a_{1}=\infty$. Let $F(z)=$ $f^{\prime}(z)-a_{2}$ and $G(z)=f(q z)-a_{2}$. Thus $F(z)$ and $G(z)$ share $0, \infty C M$. From Lemma 3.1 , we have $F(z)=k G(z)$. Combining the above with the condition that $a_{3}$ is $I M$ shared, then $k=1$, thus $f^{\prime}(z)=f(q z)$.

3.1. Remark. Theorem 3.2 is not valid for meromorphic functions with $\rho(f) \geq 1$, which can be seen by taking $f(z)=e^{z}$ and $q=-1$. We see that $f(q z)$ and $f^{\prime}(z)$ share $0,1,-1$ $C M$, but $f^{\prime}(z) \neq f(q z)$.

3.3. Theorem. Let $f(z)$ be a non-constant entire function, $q$ be a non-zero constant. If $f(q z)$ and $f^{\prime}(z)$ share two distinct constants $a, b \in \mathbb{C} C M$ and one of $a, b$ is the Picard exceptional value, then $f^{\prime}(z)=f(q z)$ or $f(z)=e^{-A z+B},-A e^{2 B}=b^{2}$ and $q=-1$.

For the proof of Theorem 3.3, we need the following three lemmas.

3.4. Lemma. [18, Theorem 1.47] Let $h(z)$ be a non-constant entire function and $f(z)=$ $e^{h(z)}$. Then $T\left(r, h^{\prime}\right)=S(r, f)$.

3.5. Lemma. [18, Theorem 1.56] Let $f_{1}, f_{2}, f_{3}$ be meromorphic functions such that $f_{1}$ is not a constant. If $f_{1}+f_{2}+f_{3}=1$ and if

$$
\sum_{j=1}^{3} N\left(r, 1 / f_{j}\right)+2 \sum_{j=1}^{3} \bar{N}\left(r, f_{j}\right)<(\lambda+o(1)) T(r),
$$

where $\lambda<1$ and $T(r):=\max _{1 \leq j \leq 3} T\left(r, f_{j}\right)$, then either $f_{2}=1$ or $f_{3}=1$.

3.6. Lemma. [7, Theorem 3.7] Let $f(z)$ be an entire function. If $f(z)$ and $f^{(l)}(z)(l \geq 2)$ have no zeros, then $f(z)=e^{A z+B}$, where $A, B$ are constants.

Proof. One of $a, b$ is the Picard exceptional value, without loss of generality, we suppose that $a$ is the Picard exceptional value. Thus

$$
f(q z)-a=e^{\alpha(z)}
$$

and

$$
f^{\prime}(z)-a=e^{\beta(z)},
$$

where $\alpha(z)$ and $\beta(z)$ are non-constant entire functions. From Lemma 3.4, we have $T\left(r, \alpha^{\prime}(z)\right)=S(r, f(q z))$. Differentiating $f(q z)$, we have

$$
f^{\prime}(q z)=\frac{1}{q} e^{\alpha(z)} \alpha^{\prime}(z)=a+e^{\beta(q z)} .
$$

From (3.1) and (3.3), we get

$$
T\left(r, e^{\alpha(z)}\right)=T(r, f(q z))+O(1) \leq T\left(r, f^{\prime}(q z)\right)+S(r, f(q z)),
$$




$$
T\left(r, e^{\beta(q z)}\right)=T\left(r, f^{\prime}(q z)\right)+O(1) .
$$

If $a \neq 0$, from (3.3), we conclude that

$$
\frac{1}{a q} e^{\alpha(z)} \alpha^{\prime}(z)-\frac{e^{\beta(q z)}}{a}=1 .
$$

Using the second main theorem for three small functions [7, Theorem 2.5], we have

$$
\begin{aligned}
T\left(r, e^{\alpha(z)}\right) & \leq \bar{N}\left(r, \frac{1}{e^{\alpha(z)}-\frac{a q}{\alpha^{\prime}(z)}}\right)+S\left(r, e^{\alpha(z)}\right) \\
& =\bar{N}\left(r, \frac{1}{e^{\beta(q z)}}\right)+S\left(r, e^{\alpha(z)}\right) \\
& =S\left(r, e^{\alpha(z)}\right),
\end{aligned}
$$

which is a contradiction. Thus, $a=0$. From (3.3), then

$$
e^{\beta(q z)-\alpha(z)}=\frac{\alpha^{\prime}(z)}{q} .
$$

Since that $b \neq 0$ is $C M$ shared by $f^{\prime}(z)$ and $f(q z)$, then we get $\frac{f^{\prime}(z)-b}{f(q z)-b}=e^{\gamma(z)}$, where $\gamma(z)$ is an entire function. Thus, combining the above with (3.1), (3.2), (3.8), we have

$$
e^{\beta(z)}-b=f^{\prime}(z)-b=e^{\gamma(z)}(f(q z)-b)=e^{\gamma(z)}\left(e^{\alpha(z)}-b\right) .
$$

Since $b \neq 0$, then

$$
\frac{e^{\beta(z)}}{b}+e^{\gamma(z)}-\frac{e^{\gamma(z)+\alpha(z)}}{b}=1 .
$$

From Lemma 3.5, if $e^{\gamma(z)} \equiv 1$, then $f^{\prime}(z)=f(q z)$ follows. If $e^{\beta(z)} \equiv b$, which implies that $f^{\prime}(z) \equiv b$, which is impossible. If $\frac{e^{\gamma(z)+\alpha(z)}}{-b} \equiv 1$, then we also have $e^{\beta(z)-\gamma(z)} \equiv-b$. Thus $e^{\alpha(z)+\beta(z)}=b^{2}$, which implies that $\alpha(z)+\beta(z) \equiv d$, where $d$ is a constant. So $\beta(q z) \equiv-\alpha(q z)+d$. Combining the above with (3.8), we have

$$
e^{-\alpha(q z)-\alpha(z)+d}=\frac{\alpha^{\prime}(z)}{q} .
$$

Remark that the left hand of (3.9) has no zeros, we have $\alpha^{\prime}(z)$ has no zeros, thus either $\alpha(z)=A z+B$ or $\alpha(z)$ is a transcendental entire function. If $\alpha(z)=A z+B$, from (3.9), we have $q=-1$ and $e^{d-2 B}=-A$. From (3.1), we have $f(z)=e^{-A z+B}$ and $-A e^{2 B}=b^{2}$. If $\alpha(z)$ is a transcendental entire function, since $\beta(z) \equiv-\alpha(z)+d$, then $\beta^{\prime}(z)$ also has no zeros. Thus from $f^{\prime \prime}(z)=\beta^{\prime}(z) e^{\beta(z)}$, then we have $f(z)$ and $f^{\prime \prime}(z)$ have no zeros, $f(z)=e^{a z+b}$ follows by Lemma 3.6, which implies that $\alpha(z)$ is a polynomial, a contradiction. Thus, we have the proof of Theorem 3.3.

3.2. Remark. If $a, b$ are not Picard exceptional values, then Theorem 3.3 is not valid, which can be seen by the function $f(z)=3 a-\frac{a}{e^{2 z}}$ and $q=-1$. Thus, $f^{\prime}(z)=\frac{2 a}{e^{2 z}}$ and $f(q z)=3 a-a e^{2 z}$ share $a$ and $2 a C M$, but $f^{\prime}(z) \neq f(q z)$.

In what follows, we will use the properties of the solutions of Fermat type equations to consider the problem that two functions share one common value. Recall the classical Fermat type equation

$$
a(z) f(z)^{n}+b(z) g(z)^{n}=1 .
$$

Yang [17, Theorem 1] obtained the following result. 
Theorem E. Let $a(z), b(z), f(z), g(z)$ be meromorphic functions, $m, n$ be positive integers. Then (3.10) can not hold, if $T(r, a(z))=S(r, f)$ and $T(r, b(z))=S(r, g)$, unless $m=n=3$. If $f(z)$ and $g(z)$ are entire, then (3.10) can not hold, even if $m=n=3$.

We get the following result, which is an improvement of [14, Corollary 1.4].

3.7. Theorem. Let $f$ be a zero-order non-constant entire function, and $q \neq 0, n \geq 2$ be an integer, and let $F=f^{n}$. If $F(z)$ and $F(q z)$ share a non-zero constant a $C M$, then $f(q z)=t f(z)$, where $t^{n}=1$.

Proof. Suppose that $F(z)$ and $F(q z)$ share a non-zero constant a CM, then we have $\frac{F(q z)-a}{F(z)-a}=C$. Thus, we have

$$
f(q z)^{n}-C f(z)^{n}=a(1-C) .
$$

If $C=1$, then we have $f(q z)=t f(z)$, where $t^{n}=1$. If $C \neq 1$, from Theorem E, we know that $n \leq 2$. From the condition $n \geq 2$, then $n=2$. In this case $f(q z)-\sqrt{c} f(z)$ and $f(q z)+\sqrt{c} f(z)$ have no zeros. Since that $f(z)$ is zero-order entire function and combining the Hadamard factorization theorem, we obtain $f(z)$ should be a constant.

3.3. Remark. (1) Theorem 3.7 is not valid for finite order entire function $f(z)$, which can be seen by taking $f(z)=e^{z}, q=-1$. Then $f(z)^{n}$ and $f(q z)^{n}$ share the value $1 \mathrm{CM}$, but $f(q z) \neq t f(z)$, where $t$ is a constant.

(2) The condition of $a \neq 0$ can not be deleted, which can be seen by $f(z)=z^{n}$ and $f(q z)=q^{n} z^{n}$ and $q^{n} \neq 1$, thus $f(z)$ and $f(q z)$ share the value $0 \mathrm{CM}$, but $f(z) \neq f(q z)$.

(3) The condition $n \geq 2$ can not be improved to $n \geq 1$, which can be seen by $f(z)=z^{n}+a$ and $q^{n}=c$, thus $\frac{f(q z)-a}{f(z)-a}=c$. Here, $f(q z)$ and $f(z)$ share the value $a$ CM, but $f(q z) \neq t f(z)$.

Brück conjecture is well-known as a classical problem in value sharing, which can be stated as follows.

Conjecture. Let $f(z)$ be a non-constant entire function, the hyper-order $\rho_{2}(f)$ is not a positive integer or infinite. If $f(z)$ and $f^{\prime}(z)$ share a finite value $b \mathrm{CM}$, then $\frac{f^{\prime}-b}{f-b}=c$, where $c$ is a non-zero constant.

The conjecture has been verified in special cases only: (1) $f$ is of finite order, see [5]; (2) $b=0$, see [3]; (3) $N\left(r, \frac{1}{f^{\prime}}\right)=S(r, f)$, see [3]. we also want to summarize some results on $q$-difference analogue of Brück conjecture.

3.8. Theorem. Let $f$ be a non-constant entire function with $\rho(f)<1$, and $q \neq 0$. If $f^{\prime}(z)$ and $f(q z)$ share a constant a $C M$, then $\frac{f^{\prime}(z)-a}{f(q z)-a}=c$.

3.4. Remark. Theorem 3.8 is easily proved. Here, we state it to show a result similar as Brück conjecture. Theorem 3.8 is not valid for finite order entire functions, which can be seen by $f(z)=e^{z}, q=-1$, thus $\frac{f^{\prime}(z)-1}{f(q z)-1}=-e^{z}$, where $f^{\prime}(z)$ and $f(q z)$ share the value $1 \mathrm{CM}$.

\section{Results on values shared by $f(q z)^{n} f^{\prime}(z)$ and $g(q z)^{n} g^{\prime}(z)$}

Hayman conjecture [6] is an important problem in the theory of value distribution. It was also considered by some authors later, such as $[2,4,13]$.

Theorem F.[4, Theorem 1] Let $f$ be a transcendental meromorphic function. If $n \geq 1$ is a positive integer, then $f(z)^{n} f^{\prime}(z)-1$ has infinitely many zeros. 
Recently, some authors investigated the zeros of $f(z)^{n} f(z+c)-a, f(z)^{n} f(q z)-a$ or their improvements, where $a$ is a non-zero constant. Some related results can be found in $[9,11,19]$. The main aim of these results is to get the sharp value of $n$ to ensure that the difference polynomials or $q$-difference polynomials admit infinitely many zeros. It is interesting to consider the value distribution of $f(q z)^{n} f^{\prime}(z)-a(z)$, where $a(z)$ is a small function with respect to $f$. We obtain the following result.

4.1. Theorem. Let $f(z)$ be a transcendental entire function with zero-order, $q \in \mathbb{C} \backslash\{0\}$ and $n \geq 1$. Then $f(q z)^{n} f^{\prime}(z)-q(z)$ has infinitely many zeros, where $q(z)$ is a non-zero polynomial.

4.2. Theorem. Let $f(z)$ be a transcendental meromorphic function with zero-order, $q \in \mathbb{C} \backslash\{0\}$ and $n \geq 9$. Then $f(q z)^{n} f^{\prime}(z)-a(z)$ has infinitely many zeros, where a $(z)$ is a non-zero small function with respect to $f(z)$.

4.1. Remark. Theorem 4.1 is not valid for finite order entire functions, which can be seen by $f(z)=e^{z}, q=-\frac{1}{n}$, and $a(z)$ is a non-constant polynomial, thus $f(q z)^{n} f^{\prime}(z)-$ $a(z)=1-a(z)$ has finitely many zeros.

For the proofs of Theorems 4.1 and 4.2, we need the following results, which were firstly considered by Barnett et al.[1], Zhang and Korhonen [19] obtained the following version.

4.3. Lemma. [19, Theorem 1.1] Let $f(z)$ be a non-constant zero-order meromorphic function and $q \in \mathbb{C} \backslash\{0\}$. Then

$$
T(r, f(q z))=T(r, f)+S(r, f)
$$

on a set of lower logarithmic density 1.

4.4. Lemma. [1, Theorem 1.1] Let $f(z)$ be a non-constant zero-order meromorphic function and $q \in \mathbb{C} \backslash\{0\}$. Then

$$
m\left(r, \frac{f(q z)}{f(z)}\right)=S(r, f)
$$

on a set of logarithmic density 1.

4.5. Lemma. Let $f(z)$ be a non-constant zero-order meromorphic function and $q \in$ $\mathbb{C} \backslash\{0\}$. Then

$$
(n-2) T(r, f) \leq T\left(r, f(q z)^{n} f^{\prime}(z)\right)+S(r, f) \leq(n+2) T(r, f)
$$

on a set of lower logarithmic density 1. If $f(z)$ is a non-constant zero-order entire function, then

$$
n T(r, f) \leq T\left(r, f(q z)^{n} f^{\prime}(z)\right)+S(r, f) \leq(n+1) T(r, f) .
$$

Proof. From Lemma 4.3 and the fact that $T\left(r, f^{\prime}(z)\right) \leq 2 T(r, f)+S(r, f)$ when $f(z)$ is a meromorphic function, we get

$$
T\left(r, f(q z)^{n} f^{\prime}(z)\right) \leq(n+2) T(r, f)+S(r, f) .
$$


Hence, the right hand side of (4.1) is true. On the other hand

$$
\begin{aligned}
(n & +1) T(r, f(z))=T\left(r, f(q z)^{n+1}\right)+S(r, f) \\
& =T\left(r, \frac{f(q z)^{n+1} f^{\prime}(z)}{f^{\prime}(z)}\right)+S(r, f) \\
& \leq T\left(r, \frac{f(q z)}{f^{\prime}(z)}\right)+T\left(r, f(q z)^{n} f^{\prime}(z)\right)+S(r, f) \\
& \leq T\left(r, \frac{f^{\prime}(z)}{f(q z)}\right)+T\left(r, f(q z)^{n} f^{\prime}(z)\right)+S(r, f) \\
& \leq N\left(r, \frac{1}{f(q z)}\right)+N\left(r, f^{\prime}(z)\right)+T\left(r, f(q z)^{n} f^{\prime}(z)\right)+S(r, f) \\
& \leq 3 T(r, f)+T\left(r, f(q z)^{n} f^{\prime}(z)\right)+S(r, f)
\end{aligned}
$$

on a set of lower logarithmic density 1. Thus, the left hand side of (4.1) is proved. If $f(z)$ is a transcendental zero-order entire function, then we have

$$
\begin{aligned}
(n+1) T(r, f(z) & =(n+1) m(r, f) \\
& \leq m\left(r, f(q z)^{n+1}\right)+S(r, f) \\
& \leq m\left(r, \frac{f(q z)}{f^{\prime}(z)}\right)+m\left(r, f(q z)^{n} f^{\prime}(z)\right)+S(r, f) \\
& \leq T\left(r, \frac{f^{\prime}(z)}{f(q z)}\right)+T\left(r, f(q z)^{n} f^{\prime}(z)\right)+S(r, f) \\
& \leq T(r, f)+T\left(r, f(q z)^{n} f^{\prime}(z)\right)+S(r, f)
\end{aligned}
$$

on a set of lower logarithmic density 1 . Combining Lemma 4.3 with the fact that $T\left(r, f^{\prime}(z)\right) \leq T(r, f)+S(r, f)$ when $f(z)$ is an entire function, we get (4.2).

Proofs of Theorems 4.1 and 4.2: Assume that $f(q z)^{n} f^{\prime}(z)-q(z)$ has only finitely many zeros, if $f(z)$ is a transcendental zero-order entire function, from Hadmard factorization theorem, we have $f(q z)^{n} f^{\prime}(z)-q(z)=p(z)$, where $p(z)$ is a non-zero polynomial. Thus, we have $n T(r, f)+S(r, f) \leq T\left(r, f(q z)^{n} f^{\prime}(z)\right)=O(\log r)$, which is impossible.

If $f(z)$ is a transcendental zero-order meromorphic function, using the second main theorem, we have

$$
\begin{aligned}
(n & -2) T(r, f(z)) \leq T\left(r, f(q z)^{n} f^{\prime}(z)\right)+S(r, f) \leq \bar{N}\left(r, f(q z)^{n} f^{\prime}(z)\right) \\
& +\bar{N}\left(r, \frac{1}{f(q z)^{n} f^{\prime}(z)}\right)+\bar{N}\left(r, \frac{1}{f(q z)^{n} f^{\prime}(z)-a(z)}\right)+S(r, f) \\
& \leq 6 T(r, f)+\bar{N}\left(r, \frac{1}{f(q z)^{n} f^{\prime}(z)-a(z)}\right)+S(r, f),
\end{aligned}
$$

which is a contradiction with $n \geq 9$.

Let $P(z)=a_{n} z^{n}+a_{n-1} z^{n-1}+\cdots+a_{1} z+a_{0}$ be a non-zero polynomial, where $a_{0}, a_{1}, \ldots, a_{n}(\neq 0)$ are complex constants and $t_{P}$ is the number of the distinct zeros of $P(z)$. The following, we will consider the generally case of $P(f(q z)) f^{\prime}(z)-a(z)$, where $a(z)$ is a small function with respect to $f(z)$.

4.6. Theorem. Let $f(z)$ be a transcendental entire function with zero-order, $q \in \mathbb{C} \backslash\{0\}$ and $n \geq 1$. Then $P(f(q z)) f^{\prime}(z)-q(z)$ has infinitely many zeros, where $q(z)$ is a non-zero polynomial.

4.7. Theorem. Let $f(z)$ be a transcendental meromorphic function with zero-order, $q \in \mathbb{C} \backslash\{0\}$ and $n \geq 2 t_{P}+7$. Then $P(f(q z)) f^{\prime}(z)-a(z)$ has infinitely many zeros, where $a(z)$ is a non-zero small function with respect to $f(z)$. 
Using the similar method as the proof of Lemma 4.5, we have the following lemma, which is needed for the proofs of Theorems 4.6 and 4.7.

4.8. Lemma. Let $f(z)$ be a non-constant zero-order meromorphic function, and $q \in$ $\mathbb{C} \backslash\{0\}$. Then

$$
(n-2) T(r, f) \leq T\left(r, P(f(q z)) f^{\prime}(z)\right)+S(r, f) \leq(n+2) T(r, f)
$$

on a set of lower logarithmic density 1 . If $f(z)$ be a non-constant zero-order entire function,

$$
n T(r, f) \leq T\left(r, P(f(q z)) f^{\prime}(z)\right)+S(r, f) \leq(n+1) T(r, f)
$$

on a set of lower logarithmic density 1.

Finally, we consider the uniqueness of $f(q z)^{n} f^{\prime}(z)$ and $g(q z)^{n} g^{\prime}(z)$ sharing a non-zero polynomial and obtain the following result.

4.9. Theorem. Let $f(z)$ and $g(z)$ be transcendental entire functions with zero-order, $q \in \mathbb{C} \backslash\{0\}$ and $n \geq 5$. If $f(q z)^{n} f^{\prime}(z)$ and $g(q z)^{n} g^{\prime}(z)$ share a non-zero polynomial $p(z)$ $C M$, then we have $f(q z)^{n} f^{\prime}(z)=g(q z)^{n} g^{\prime}(z)$.

Proof. From the conditions, we get $\frac{f(q z)^{n} f^{\prime}(z)-p(z)}{g(q z)^{n} g^{\prime}(z)-p(z)}=c$. If $c=1$, then $f(q z)^{n} f^{\prime}(z)=$ $g(q z)^{n} g^{\prime}(z)$ follows. If $c \neq 1$, then we have

$$
f(q z)^{n} f^{\prime}(z)-c g(q z)^{n} g^{\prime}(z)=p(z)(1-c) .
$$

Using the second main theorem, we get

$$
\begin{aligned}
& T\left(r, f(q z)^{n} f^{\prime}(z)\right) \leq \bar{N}\left(r, f(q z)^{n} f^{\prime}(z)\right)+\bar{N}\left(r, \frac{1}{f(q z)^{n} f^{\prime}(z)}\right) \\
+ & \bar{N}\left(r, \frac{1}{f(q z)^{n} f^{\prime}(z)-(1-c) p(z)}\right)+S\left(r, f(q z)^{n} f^{\prime}(z)\right) \\
\leq & N\left(r, \frac{1}{f(q z)}\right)+N\left(r, \frac{1}{f^{\prime}(z)}\right)+\bar{N}\left(r, \frac{1}{g(q z)^{n} g^{\prime}(z)}\right)+S(r, f) \\
\leq & 2 T(r, f)+2 T(r, g)+S(r, f) .
\end{aligned}
$$

Similar as the above, we also get

$$
T\left(r, g(q z)^{n} g^{\prime}(z)\right) \leq 2 T(r, f)+2 T(r, g)+S(r, g) .
$$

Combining (4.9), (4.10) with (4.2), we have

$$
n[T(r, f)+T(r, g)] \leq 4[T(r, f)+T(r, g)]+S(r, f)+S(r, g),
$$

which is a contradiction with the condition $n \geq 5$.

Acknowledgment. The authors would like to thank the referee for his/her helpful suggestions and comments. This work was partially supported by the NSFC (No. 11301260, 11461042).

\section{References}

[1] Barnett, D., Halburd, R.G., Korhonen, R.J., Morgan, W. Applications of Nevanlinna theory to q-difference equations, Proc. Roy. Soc. Edinburgh Sect. A, 137 457-474, 2007.

[2] Bergweiler, W. and Eremenko, A. On the singularities of the inverse to a meromorphic function of finite order, Revista Matemática Iberoamericana 11, 355-373, 1995.

[3] Brück, R. On entire functions which share one value CM with their first derivative, Result. Math 30, 21-24, 1996.

[4] Chen, H.H. and Fang, M.L. On the value distribution of $f^{n} f^{\prime}$, Sci. China Ser. A 38, 789-798, 1995. 
[5] Gundersen, G.G. and Yang, L.Z. Entire functions that share one value with one or two of their derivatives, J. Math. Anal. Appl 223, 88-95, 1998.

[6] Hayman, W.K. Picard values of meromorphic functions and their derivatives, Ann. Math 70, 9-42, 1959

[7] Hayman, W.K. Meromorphic Functions. Oxford at the Clarendon Press, 1964.

[8] Heittokangas, J., Korhonen, R.J., Laine. I., Rieppo, J., Zhang, J.L. Value sharing results for shifts of meromorphic function, and sufficient conditions for periodicity, J. Math. Anal. Appl 355, 352-363, 2009.

[9] Laine, I. and Yang, C.C. Value distribution of difference polynomials, Proc. Japan Acad. Ser. A 83, 148-151, 2007.

[10] Laine, I. Nevanlinna Theory and Complex Differential Equations. Walter de Gruyter, BerlinNew York, 1993.

[11] Liu, K., Cao, T.B., Liu, X.L. Some difference results on Hayman conjecture and uniqueness, Bull. Iranian. Math. Soc 38 (4), 1007-1020, 2012.

[12] Mues, E. and Steinmetz, N. Meromorphe funktionen, die mit ihrer ableitung werte teilen, Manuscripta Math 29, 195-206, 1979.

[13] Mues, E., Über ein Problem von Hayman, Math. Z 164, 239-259, 1979.

[14] Qi, X.G., Liu, K., Yang, L.Z. Value sharing results of a meromorphic function $f(z)$ and $f(q z)$, Bull. Korean Math. Soc 48 (6), 1235-1243, 2011.

[15] Nevanlinna, R. Le théorème de Picard-Borel et la théorie des fonctions méromorphes, Gauthiers-Villars, Paris, 1929.

[16] Rubel, L. and Yang, C.C. Values shared by an entire function and its derivative, in Complex Analysis, Kentucky, 1976, Lecture Notes in Math., vol. 599, Springer-Verlag, Berlin, 101-103, 1977.

[17] Yang, C.C. A generalization of a theorem of P. Montel on entire functions, Proc. Amer. Math. Soc 26, 332-334, 1970.

[18] Yang, C.C. and Yi, H.X. Uniqueness Theory of Meromorphic Functions, Kluwer Academic Publishers, 2003.

[19] Zhang, J.L. and Korhonen, R.J. On the Nevanlinna characteristic of $f(q z)$ and its applications, J. Math. Anal. Appl 369, 537-544, 2010. 